Raterman, H.G., Nielen, M.M.J., Peters, M.J.L., Verheij, R.A., Nurmohamed, M.T., Schellevis, F.G. Coexistence of hypothyroidism with inflammatory arthritis is associated with cardiovascular disease in women. Annals of the Rheumatic Diseases: 2012, 71(7), 1216-1218

\begin{tabular}{|l|l|}
\hline Postprint Version & 1.0 \\
\hline Journal website & $\underline{\text { http://ard.bmj.com/content/early/2012/03/13/annrheumdis-2011-200836.long }}$ \\
\hline Pubmed link & $\underline{\text { http://www.ncbi.nlm.nih.gov/pubmed/22419774 }}$ \\
\hline DOI & $10.1136 /$ annrheumdis-2011-200836 \\
\hline
\end{tabular}

This is a NIVEL certified Post Print, more info at http://www.nivel.eu

\title{
Coexistence of hypothyroidism with inflammatory arthritis is associated with cardiovascular disease in women.
}

\author{
Hennie G Raterman ${ }^{1}$, MARK M J Nielen ${ }^{2}$, Mike J L Peters ${ }^{3}$, Robert A VerheiJ ${ }^{2}$, Michael T \\ NURMOHAMED ${ }^{3,4}$, FRANCOIS G SCHELLEVIS ${ }^{2,5}$ \\ 1Department of Rheumatology, VU University Medical Center, Amsterdam, Netherlands \\ 2Netherlands Institute for Health Services Research, Utrecht, Netherlands \\ 3Department of Internal Medicine, VU University Medical Center, Amsterdam, Netherlands \\ 4Department of Rheumatology, Jan van Breemen Research Institute/Reade, Amsterdam, Netherlands \\ 5General Practice, EMGO Institute for Health and Care Research, VU University Medical Center, \\ Amsterdam, Netherlands
}

\begin{abstract}
Objectives Hypothyroidism and inflammatory arthritis tend to coexist, but data on this association are sparse. In terms of cardiovascular risk, this association may have clinical relevance as this coexistence may carry an additional cardiovascular risk. This study calculates, first, the prevalence of hypothyroidism in patients with inflammatory arthritis and, second, the cardiovascular disease (CVD) prevalence rate in patients with either hypothyroidism or inflammatory arthritis, or both.

Methods Data from the Netherlands Information Network of General Practice, a representative Dutch sample of 360000 registered patients, were used. Prevalence rates of hypothyroidism were calculated, and multilevel logistic regression analyses were used to calculate CVD prevalence rates.

Results Hypothyroidism prevalence was $6.5 \%$ in female patients with arthritis compared to $3.9 \%$ in controls $(\mathrm{p}<0.001)$. CVD prevalence was $4.3 \%$ in patients with hypothyroidism, $5.9 \%$ in patients with inflammatory arthritis, $14.3 \%$ in patients with hypothyroid inflammatory arthritis and 2.1\% in controls. Adjusted CVD prevalence rates were 1.2 (95\% CI 0.99 to 1.4) for hypothyroidism, 1.5 (95\% CI 1.1 to 2.0) for inflammatory arthritis and 3.7 (95\% CI 1.7 to 8.0) for hypothyroid inflammatory arthritis as compared with controls.

Conclusions These data raise awareness on the coexistence of hypothyroidism and inflammatory arthritis and emphasise the importance of cardiovascular risk management in these patients, particularly when hypothyroidism and inflammatory arthritis coexist.
\end{abstract}

\section{INTRODUCTION}

Autoimmune disorders are disease entities with a broad spectrum of manifestations, as the manifestations are, on one hand, more organ specific (such as autoimmune thyroiditis) and, on the other hand, more systemic (such as several rheumatic disorders). Traditionally, autoimmune diseases were considered separate disorders. However, recently, it has become clear that autoimmune diseases share similarities in genetic and immunological origins and environmental risk factors, suggesting that autoimmune diseases 
Raterman, H.G., Nielen, M.M.J., Peters, M.J.L., Verheij, R.A., Nurmohamed, M.T., Schellevis, F.G. Coexistence of hypothyroidism with inflammatory arthritis is associated with cardiovascular disease in women. Annals of the Rheumatic Diseases: 2012, 71(7), 1216-1218

cluster. ${ }^{12}$ Autoimmune thyroiditis (the most prevalent autoimmune disorder) and inflammatory arthritis appear to be associated, but data on this relationship are sparse. ${ }^{34}$

In addition to their coexistence, both hypothyroidism and inflammatory arthritis are associated with a higher cardiovascular disease (CVD) burden. ${ }^{5},{ }^{-7}$ Consequently, a question arises on whether the clustering of hypothyroidism and inflammatory arthritis is associated with an amplified CVD prevalence. As most of the studies on CVD risk and autoimmune conditions were performed on patients with arthritis from referral centres and as the CVD risk of these patients may not reflect or may overestimate the actual cardiovascular risk of patients in the general population, it is important to assess cardiovascular risk in primary care patients as a more representative sample of the general population.

The aims of the present study were to ascertain the prevalence of hypothyroidism in patients with inflammatory arthritis compared to patients with non-inflammatory arthritis and to determine CVD prevalence rates in patients with either hypothyroidism or inflammatory arthritis, or both, as compared with control subjects.

\section{METHODS}

\section{Study population}

Data from the Netherlands Information Network of General Practice were used. These data were retrieved from the electronic medical records of a representative sample of 69 general practices with 360000 registered patients in 2006. Data included information on consultations, morbidity, prescriptions and referrals to other healthcare professionals. The patients and general practices are representative of the Dutch population. ${ }^{8}$ Patients below 30 years old were excluded because of their lower probability of having inflammatory arthritis, hypothyroidism and/or CVD. Practices that recorded data for less than 6 months were excluded from further analysis. The study was carried out in accordance with Dutch legislation on privacy. The privacy regulation of the study was approved by the Dutch Data Protection Authority. According to Dutch legislation, obtaining informed consent or approval by a medical ethics committee was not obligatory for observational studies.

\section{Classification of diagnoses}

Morbidity data were derived from consultation diagnoses and, furthermore, from all prescriptions issued by the participating practices. Diagnoses were recorded using the International Classification of Primary Care (ICPC)-1 coding system. ${ }^{9}$ When a prescription is issued, a diagnostic code is recorded, and the selected drug is automatically linked to the Anatomical Therapeutic Chemical (ATC) Classification System. ${ }^{10}$ This study used the combination of ATC and ICPC- 1 code to determine whether the patient was diagnosed as having inflammatory arthritis (ICPC code L88 (rheumatoid arthritis (RA) or ankylosing spondylitis)), hypothyroidism (ICPC code T86 and/or ATC code H03AA01 (levothyroxine)) and CVD (ICPC code K75 (myocardial infarction), K89 (transient ischemic attack) and/or K90

(stroke/cerebrovascular accident)) in 2006 or in the years before.

\section{Statistical analyses}

In a first analysis, the prevalence rate of hypothyroidism in persons with and without inflammatory arthritis (reference group) was calculated for men and women separately in several age groups. Differences in the prevalence rates of hypothyroidism between patients with inflammatory arthritis and the reference group were tested with chi-square tests.

The prevalence rates of CVD in either inflammatory arthritis or hypothyroidism were compared with multilevel logistic regression analyses with a random intercept using the second-order PQL (penalized quasi-likelihood) method. All patients were categorised into inflammatory arthritis without hypothyroidism, hypothyroidism without inflammatory arthritis, inflammatory arthritis and hypothyroidism, or controls (reference group). This categorical variable was added to the model with the control category as reference. The association of inflammatory arthritis and/or hypothyroidism with CVD was adjusted for risk factors: age, hypertension (ICPC code K86 and/or K87), hypercholesterolemia (ICPC code T93) and diabetes (ICPC code T90 and/or ATC code A10). We restricted the analyses to female subjects, as there were too few men with both hypothyroidism and inflammatory arthritis to yield meaningful estimates. Chi-square tests were performed with Stata10. Multilevel analyses were performed with MLwiN, a statistical program for multilevel analyses. 
Raterman, H.G., Nielen, M.M.J., Peters, M.J.L., Verheij, R.A., Nurmohamed, M.T., Schellevis, F.G. Coexistence of hypothyroidism with inflammatory arthritis is associated with cardiovascular disease in women. Annals of the Rheumatic Diseases: 2012, 71(7), 1216-1218

\section{RESULTS}

\section{Hypothyroidism prevalence}

In total, 175061 subjects were studied: 1518 (0.9\%) of these subjects were patients with inflammatory arthritis. In both male and female patients with inflammatory arthritis, hypothyroidism prevalence rates were significantly higher than in controls: $2.4 \%$ versus $0.8 \%$ in male patients and $6.5 \%$ versus $3.9 \%$ in female patients (table 1).

\section{[TABLE 1$]$}

\section{CVD prevalence rates}

Crude CVD prevalence rates were $4.4 \%$ in patients with hypothyroidism, 5.9\% in patients with inflammatory arthritis and $14.3 \%$ in patients with hypothyroid inflammatory arthritis, as compared with $2.2 \%$ in control subjects. Multilevel analyses revealed significantly higher ORs for all groups as compared with control subjects (table 2). Adjustment for age, hypertension, diabetes mellitus and hypercholesterolemia moderately attenuated these OR, but inflammatory arthritis and hypothyroid inflammatory arthritis remained significantly associated with CVD (model II, table 2). Testing for effect modification showed no differences between those above 50 years of age and those below 50 years of age.

\section{[TABLE 2 ]}

\section{DISCUSSION}

This study demonstrated a doubled prevalence of hypothyroidism in patients with inflammatory arthritis from the general population as compared to controls. This is an important observation given the association of both hypothyroidism and inflammatory arthritis with CVD, which is amplified when hypothyroidism and inflammatory arthritis coexist.

Decades ago, signs of hypothyroidism had been reported in $12-30 \%$ of patients with arthritis; therefore, it seems likely that the prevalence rates of hypothyroidism are increased in patients with inflammatory arthritis. ${ }^{11} 12$ Indeed, we observed an increased prevalence of hypothyroidism in patients with inflammatory arthritis when compared to controls. These results emphasise the tendency of autoimmune disorders to cluster. ${ }^{13}$ Explanations for the coexistence of autoimmune disorders involve immunological disturbances (in $\mathrm{B}$ and $\mathrm{T}$ lymphocytes), a tendency to react abnormally in the presence of an antigen or genetic susceptibility. ${ }^{13} 14$

Inflammatory arthritis and hypothyroidism are independently associated with a higher CVD risk. Thyroid hormone deficiency is known to deteriorate endothelial dysfunction directly by increasing oxidative stress, decreasing nitric oxide production and increasing platelet activation, thereby stimulating atherogenesis. ${ }^{15}$ Furthermore, hypothyroidism is associated with (subclinical) atherosclerosis in cardiovascular-event-naive female patients with RA. ${ }^{16}{ }^{17}$ Moreover, we previously described an increased cardiovascular risk in CVDevent-naïve female patients with hypothyroid RA, as reflected by a higher Framingham score and a higher prevalence of metabolic syndrome compared to euthyroid female patients with RA. ${ }^{18}$ In the present study, CVD prevalence rates were significantly higher in female patients with hypothyroid inflammatory arthritis as compared to either patients with inflammatory arthritis alone or patients with hypothyroidism alone. This observation in primary care patients (as a more representative sample of the general population) confirms previous results in female secondary care patients with RA, indicating a clear association between hypothyroidism and CVD. ${ }^{19}$ In this respect, it is noteworthy that functional polymorphisms of protein tyrosine phosphatase N22 - a susceptibility factor for several autoimmune diseases such as hypothyroidism, inflammatory arthritis and diabetes - accelerate atherosclerosis. ${ }^{20}$

Limitations of this study merit careful consideration. First, causality cannot be shown in this crosssectional case-control study. Second, several CVD risk factors, such as lifestyle factors, family history of CVD, socioeconomic status and ethnic background, were unavailable and could not be adjusted for in this study. Third, we cannot exclude that part of our observed findings may be explained by an increased frequency of testing for thyroid disorders, as we and others previously described an increased prevalence of hypothyroidism in secondary care patients with RA. On the other hand, this study describes for the first time the association between hypothyroidism and arthritis in the general population; in clinical practice, 
Raterman, H.G., Nielen, M.M.J., Peters, M.J.L., Verheij, R.A., Nurmohamed, M.T., Schellevis, F.G. Coexistence of hypothyroidism with inflammatory arthritis is associated with cardiovascular disease in women. Annals of the Rheumatic Diseases: 2012, 71(7), 1216-1218

testing more frequently for thyroid disorders is not widely adopted. Fourth, the ICPC coding system was not developed for research purposes and does not differentiate between different inflammatory arthritis types, that is, rheumatoid and psoriatic arthritis or ankylosing spondylitis. Moreover, we cannot exclude that certain patients in the reference group included patients with arthritis. However, in this case, misclassification would more likely increase the strength of our findings than weaken it. Nevertheless, the results of this study concern a large representative sample of the Dutch general population and may be the best source for a more precise estimate of cardiovascular burden, as the general practitioner acts as a gatekeeper for referral to specialised care in the general population. ${ }^{8}$ Previous studies comprised mostly secondary care patients with arthritis with a possibly more severe disease; therefore, the CVD risk of these patients may not reflect or may overestimate the actual cardiovascular risk in the general population. As our study observes a clear association between hypothyroidism, inflammatory arthritis and CVD in primary care patients, this association needs further elaboration in prospective studies.

In conclusion, our observations raise awareness on the coexistence of autoimmune disorders ('polyautoimmune disorder syndromes') as the prevalence of hypothyroidism is twofold elevated compared to control subjects. This is a clinically important observation, given the substantially elevated CVD prevalence rates when hypothyroidism and inflammatory arthritis coexist, and emphasises the need for cardiovascular risk management in this case.

\section{FOOTNOTES}

Competing interests None.

Provenance and peer review Not commissioned; externally peer reviewed.

\section{REFERENCES}

1. Bach JF. The effect of infections on susceptibility to autoimmune and allergic diseases. N Engl J Med 2002;347:911-20.

2. Anaya JM, Corena R, Castiblanco J, et al. The kaleidoscope of autoimmunity: multiple autoimmune syndromes and familial autoimmunity. Expert Rev Clin Immunol 2007;3:623-35.

3. Lazúrová I, Benhatchi K, Rovenský J, et al. Autoimmune thyroid disease and autoimmune rheumatic disorders: a two-sided analysis. Ann N Y Acad Sci 2009;1173:211-6.

4. Somers EC, Thomas SL, Smeeth L, et al. Are individuals with an autoimmune disease at higher risk of a second autoimmune disorder? Am J Epidemiol 2009;169:749-55.

5. Solomon DH, Karlson EW, Rimm EB, et al. Cardiovascular morbidity and mortality in women diagnosed with rheumatoid arthritis. Circulation 2003;107:1303-7.

6. Peters MJ, van Halm VP, Voskuyl AE, et al. Does rheumatoid arthritis equal diabetes mellitus as an independent risk factor for cardiovascular disease? A prospective study. Arthritis Rheum 2009;61:1571-9.

7. Hak AE, Pols HA, Visser TJ, et al. Subclinical hypothyroidism is an independent risk factor for atherosclerosis and myocardial infarction in elderly women: the Rotterdam Study. Ann Intern Med 2000;132:270-8.

8. Fatehi D, van der Zee J, van der Wal E, et al. Temperature data analysis for 22 patients with advanced cervical carcinoma treated in Rotterdam using radiotherapy, hyperthermia and chemotherapy: a reference point is needed. Int J Hyperthermia 2006;22:353-63.

9. Lamberts H, Wood M. International Classification of Primary Care. Oxford: Oxford University Press, 1987.

10. ATC/DDD Index 2011. http://www.whocc.no/atc_ddd_index/. 2011. (Accessed 1 October 2011).

11. Hart FD. Rheumatoid arthritis: extra-articular manifestations. II. BMJ 1970;2:747-52.

12. Shiroky JB, Cohen M, Ballachey ML, et al

13. . Thyroid dysfunction in rheumatoid arthritis: a controlled prospective survey. Ann Rheum Dis 1993;52:454-6.

14. Eisenbarth GS, Gottlieb PA. Autoimmune polyendocrine syndromes. N Engl J Med 2004;350:2068-79.

15. Laivoranta-Nyman S, Möttönen T, Hermann R, et al. HLA-DR-DQ haplotypes and genotypes in Finnish patients with rheumatoid arthritis. Ann Rheum Dis 2004;63:1406-12.

16. van Zaane B, Reuwer AQ, Büller HR, et al. Hormones and cardiovascular disease: a shift in paradigm with clinical consequences? Semin Thromb Hemost 2009;35:478-87. 
Raterman, H.G., Nielen, M.M.J., Peters, M.J.L., Verheij, R.A., Nurmohamed, M.T., Schellevis, F.G. Coexistence of hypothyroidism with inflammatory arthritis is associated with cardiovascular disease in women. Annals of the Rheumatic Diseases: 2012, 71(7), 1216-1218

17. Schott LL, Kao AH, Cunningham A, et al. Do carotid artery diameters manifest early evidence of atherosclerosis in women with rheumatoid arthritis? J Womens Health (Larchmt) 2009;18:21-9.

18. Dessein PH, Norton GR, Woodiwiss AJ, et al. Influence of nonclassical cardiovascular risk factors on the accuracy of predicting subclinical atherosclerosis in rheumatoid arthritis. J Rheumatol 2007;34:943-51.

19. Raterman HG, van Eijk IC, Voskuyl AE, et al. The metabolic syndrome is amplified in hypothyroid rheumatoid arthritis patients: a cross-sectional study. Ann Rheum Dis 2010;69:39-42.

20. Raterman HG, van Halm VP, Voskuyl AE, et al. Rheumatoid arthritis is associated with a high prevalence of hypothyroidism that amplifies its cardiovascular risk. Ann Rheum Dis 2008;67:229 32.

21. Pertovaara M, Raitala A, Juonala M, et al. Autoimmunity and atherosclerosis: functional polymorphism of PTPN22 is associated with phenotypes related to the risk of atherosclerosis. The Cardiovascular Risk in Young Finns Study. Clin Exp Immunol 2007;147:265-9.

\section{TABLES}

Table 1 Prevalence of hypothyroidism in patients with inflammatory arthritis and in controls

\begin{tabular}{|c|c|c|c|c|c|c|c|}
\hline & \multicolumn{3}{|c|}{ Inflammatory arthritis } & \multicolumn{3}{|c|}{ Controls } & \multirow[b]{2}{*}{ p Value } \\
\hline & $n$ & Total & Prevalence rate $(\%)$ & $n$ & Total & Prevalence rate $(\%)$ & \\
\hline Total & 76 & 1518 & 5.01 & 4.153 & 173543 & 2.39 & $<0.00050$ \\
\hline \multicolumn{8}{|l|}{ Gender } \\
\hline Male & 13 & 545 & 2.39 & 681 & 85405 & 0.80 & $<0.00050$ \\
\hline Female & 63 & 973 & 6.47 & 3.471 & 88126 & 3.94 & $<0.00050$ \\
\hline \multicolumn{8}{|c|}{ Age (years) } \\
\hline $30-39$ & 4 & 128 & 3.13 & 422 & 40985 & 1.03 & 0.02 \\
\hline $40-49$ & 2 & 253 & 0.79 & 714 & 42543 & 1.68 & 0.27 \\
\hline 50-59 & 16 & 369 & 4.34 & 951 & 37466 & 2.54 & 0.03 \\
\hline $60-69$ & 21 & 318 & 6.60 & 854 & 25919 & 3.29 & 0.001 \\
\hline $70-79$ & 20 & 274 & 7.30 & 740 & 16956 & 4.36 & 0.02 \\
\hline$\geq 80$ & 13 & 176 & 7.39 & 472 & 9665 & 4.88 & 0.13 \\
\hline
\end{tabular}

Table 2 Prevalence odds ratios for CVD in patients with hypothyroidism and inflammatory arthritis

\begin{tabular}{|c|c|c|}
\hline & $\begin{array}{l}\text { Model 1* OR } \\
(95 \% \mathrm{Cl})\end{array}$ & $\begin{array}{l}\text { Model 2+ OR } \\
(95 \% \mathrm{CI})\end{array}$ \\
\hline Controls $(n=84655)$ & 1.0 & 1.0 \\
\hline Hypothyroidism $(n=3471)$ & $1.94(1.63$ to 2.31$) \ddagger$ & $1.19(0.99$ to 1.43$)$ \\
\hline Inflammatory arthritis $(n=910)$ & $2.44(1.83$ to 3.24$) \ddagger$ & $1.48(1.10$ to 2.00$) \ddagger$ \\
\hline $\begin{array}{l}\text { Hypothyroidism and inflammatory } \\
\text { arthritis }(n=63)\end{array}$ & $6.38(3.10$ to 13.12$) \ddagger$ & $3.72(1.74$ to 7.95$) \ddagger$ \\
\hline
\end{tabular}

*Logistic multilevel multivariate regression analyses with CVD as outcome variable: crude analysis.

†Adjustment for age, hypertension, hypercholesterolemia and diabetes.

$\ddagger \mathrm{p}<0.05$.

CVD, cardiovascular disease. 